\title{
Diseño de un sistema de apoyo a la regulación social del aprendizaje en los XMOOC
}

\author{
Design of a Support System for the Social Regulation of Learning in XMOOC \\ Disseny d'un sistema de suport a la regulació social de l'aprenentatge en els xMOOC
}

\author{
Iolanda Garcia $^{\circledR}$, Elena Barberà ${ }^{\circledR}$, Marcelo Maina ${ }^{\circledR}$ \\ Universitat Oberta de Catalunya
}

Recibido: 11/10/2019 | Aceptado: 09/12/2019 | Publicado: 20/12/2019

Cómo citar: Garcia, I.; Barberà, E.; y Maina, M. (2019). Diseño de un sistema de apoyo a la regulación social del aprendizaje en los XMOOC. Research in Education and Learning Innovation Archives, 23,43-61. 10.7203/realia.23.15914

Copyright: El/La Autor/a. Open Access: Este es un artículo de acceso abierto distribuido bajo los términos de la licencia Creative Commons AttributionNonCommercial-ShareAlike 4.0 International (CC BY-NC-SA 4.0)

Financiación: Este trabajo forma parte del proyecto de investigación REGinNET (EDU2016-76434-P),

financiado dentro del Plan Nacional de I+D del Ministerio de Ciencia, Innovación y Universidades de España.

\begin{abstract}
RESUMEN: Esta investigación tiene como objetivo estudiar los procesos de regulación del aprendizaje, atendiendo tanto a su dimensión individual como social, en entornos de xMOOC. En concreto, se propone analizar cómo los procesos de autorregulación, corregulación y regulación social compartida pueden apoyarse y promoverse en este tipo de contextos abiertos y masivos para conseguir un aprendizaje más profundo. Para ello, se aplica un modelo de investigación basada en el diseño que permite intervenir directamente en la práctica pedagógica, a través de un proceso cíclico de cuatro etapas: diseño, intervención, evaluación y reflexión y rediseño de un sistema de apoyo a la regulación del aprendizaje en un xMOOC. En este artículo se presenta la primera iteración de la investigación junto con los resultados de las entrevistas semiestructuradas con algunos participantes. En primer lugar, se realiza una revisión de literatura de la que derivan los fundamentos teóricos del diseño aplicados en el sistema de apoyo a la regulación planteado. A continuación, se describe con detalle el diseño e implementación de dicho sistema conformado por un conjunto de avisos (prompts) introducidos de manera estratégica en un xMOOC sobre gamificación. Seguidamente se discuten los resultados obtenidos, entre los que destacan el diverso aprovechamiento de los tipos de avisospor parte de los participantes y la dificultad de activar indicadores de orientación y reflexión de carácter social, en un entorno de aprendizaje de las características de un xMOOC. Finalmente se extraen algunas conclusiones y recomendaciones para el diseño de la regulación y la práctica de aprendizaje en XMOOC.
\end{abstract}

PALABRAS CLAVE: Diseño del aprendizaje; regulación del aprendizaje; autorregulación; corregulación; regulación social compartida; MOOC

ABSTRACT: This research aims at studying the processes of learning regulation in xMOOC environments, attending to both the individual and social dimensions of learning. Specifically, it attempts to analyze how the processes of self-regulation, co-regulation and socially shared regulation can be supported and promoted in open and massive learning contexts to achieve a deeper learning. To this end, a design-based research model is applied to allow a direct intervention in pedagogical practice, through a cyclic process of four stages: design, intervention, evaluation and reflection and redesign of a support system for the regulation of learning in an XMOOC. This paper presents the first iteration of the research together with the results of semi-structured interviews with some participants. First, we present the theoretical foundations of the design that have been applied to the proposed regulation support system as a result of a comprehensive literatura review. Next, we describe the implementation of the system, composed of a set of prompts strategically administered into an xMOOC on gamification. Then, we present the results, among which we highlight the diverse use of the prompts made by the participants and the challenges to activate traces of social orientation and reflection in a learning environment of the characteristics of an xMOOC. Finally, some conclusions 
and recommendations for the design of learning regulation and practice in $\mathrm{xMOOC}$ are drawn.

KEYWORDS: Learning design; learning regulation; self-regulation; co-regulation; socially shared regulation; MOOC

RESUM: Aquesta investigació té com a objectiu estudiar els processos de regulació de l'aprenentatge, atenent tant a la seva dimensió individual com social, en entorns de xMOOC. En concret, es proposa analitzar com els processos d'autoregulació, coregulació i regulació social compartida poden recolzar-se i promoure's en aquest tipus de contextos oberts i massius per tal d'aconseguir un aprenentatge més profund. Per a això, s'aplica un model d'investigació basada en el disseny que permet intervenir directament en la pràctica pedagògica, a través d'un procés cíclic de quatre etapes: disseny, intervenció, avaluació i reflexió i redisseny d'un sistema de suport a la regulació de l'aprenentatge en un xMOOC. En aquest article es presenta la primera iteració de la investigació junt amb els resultats de les entrevistes semiestructurades amb alguns participants. En primer lloc, es realitza una revisió de literatura de la qual deriven els fonaments teòrics del disseny aplicats en el sistema de suport a la regulació plantejat. A continuació, es descriu amb detall el disseny i la implementació d'aquest sistema conformat per un conjunt d'avisos (prompts) introduits de manera estratègica en un xMOOC sobre gamificació. Seguidament es discuteixen els resultats obtinguts, entre els quals destaquen el divers aprofitament dels diferents avisosper part dels participants i la dificultat d'activar indicadors d'orientació i reflexió de caràcter social, en un entorn d'aprenentatge de les característiques d'un xMOOC. Finalment s'extreuen algunes conclusions i recomanacions per al disseny de la regulació i la pràctica de l'aprenentatge en xMOOC.

PARAULES CLAU: Disseny de l'aprenentatge; regulació de l'aprenentatge; autoregulació; coregulació; regulació social compartida; MOOC

\section{Notas de aplicación práctica}

\section{Qué se sabe sobre este tema}

- El diseño tecnopedagógico de cada MOOC determina el tipo de experiencia de aprendizaje de los participantes y también su nivel de calidad en términos instruccionales.

- Un aprovechamiento formativo óptimo en los MOOC requiere poner en juego estrategias de regulación del aprendizaje que no todos los participantes necesariamente han desarrollado o saben emplear correctamente.

- Actualmente no existen propuestas para el apoyo de los procesos de regulación del aprendizaje en MOOC en un sentido global, es decir, incluyendo los procesos de autorregulación, de corregulación y de regulación social compartida.

\section{Qué aporta este trabajo}

- Una propuesta de apoyo de los procesos de regulación individual y social en los xMOOC desde el campo del diseño del aprendizaje basada en el uso de prompts.

- Recomendaciones generales para la mejora del diseño del aprendizaje en los xMOOC.

Implicaciones para la práctica y/o política

- Las motivaciones y la aproximación al aprendizaje iniciales en el MOOC por parte de los participantes determina y condiciona el uso y aprovechamiento de un sistema de ayuda a la regulación del aprendizaje.

- La implementación de un sistema de apoyo a la regulación del aprendizaje en la plataforma tecnológica de los xMOOC debe ser transparente para los participantes, integrarse al máximo en los contenidos del curso, y a la vez trazar un itinerario reconocible y comprensible.

- Para la implementación de un sistema de apoyo a la regulación social del aprendizaje es indispensable crear las condiciones necesarias en el diseño del aprendizaje del MOOC, que garanticen el desarrollo de actividades que impliquen procesos de interacción social y de colaboración entre los participantes. 


\section{INTRODUCCIÓN}

En su conceptualización inicial los MOOC materializaron los principios del conectivismo y de la educación abierta, como entornos en red en que las personas interactúan entre sí y con objetos de conocimiento, aprendiendo a través de estas conexiones (Anderson y McGreal, 2012; Powell y Yuan, 2013). Este tipo de MOOC, inspirado en el modelo conectivista, fue denominado cMOOC. Más tarde surgieron los llamados xMOOC, actualmente los más generalizados, que enfatizan el aprendizaje individual a través de materiales interactivos en lugar del aprendizaje social, como sería el caso de los cMOOC (Conole, 2015; Daniel, 2012; Guàrdia, Maina, y Sangrà, 2013). Coincidimos con Conole (2015) en que la distinción entre cMOOC y xMOOC es excesivamente reduccionista y limitada. De hecho, atendiendo al enfoque pedagógico utilizado podríamos identificar diferentes modelos de MOOC.

Desde su aparición, numerosos autores han señalado diversos problemas de los MOOC, tales como: su calidad cuestionable desde el punto de vista tecnopedagógico, su baja tasa de finalización y alta tasas de abandono, validez de la certificación y la acreditación, entre otros. Según el análisis de Margaryan, Bianco, y Littlejohn (2015), la mayoría de los MOOC tienen una baja calidad en cuanto a su diseño instruccional, por ejemplo, al no contemplar suficientemente el apoyo en los procesos de interacción y de retroalimentación (Clarà y Barbera, 2013; Clarà y Barberà, 2014) .

Así, distintas propuestas han aparecido en los últimos años para compensar las deficiencias observadas. El hMOOC, por ejemplo, es un modelo híbrido propuesto por Fidalgo-Blanco, Sein-Echaluce, y García-Peñalvo (2016) que combina el uso de las plataformas prototípicas de xMOOC y cMOOC (LMS y redes sociales). De esta forma, propone la realización de actividades estructuradas y formalizadas, junto con otras de tipo informal y de cooperación entre los participantes, con la intención de generar un flujo y retroalimentación constante entre ambos procesos. Más tarde, el modelo ahMOOC propone incorporar además herramientas adaptativas que permitan a los participantes elegir entre ciertos recursos y actividades, con el propósito de personalizar el itinerario de aprendizaje en cada caso (García-Peñalvo, Fidalgo-Blanco, y Sein-Echaluce, 2018). Los denominados sMOOC, "s" de "social" y de seamless, por el hecho de potenciar, por una parte, la interacción en el aprendizaje y por otra, la característica de ubicuidad de los cursos, que posibilita su acceso desde cualquier dispositivo, en cualquier lugar y momento (sin costuras o seamless). Los tMOOC tienen como objetivo central la transferencia del aprendizaje y la transformación pedagógica, orientándose hacia la acción y la interacción profesional. Estos dos últimos modelos se desarrollaron dentro del Proyecto Europeo ECO, en que los participantes del MOOC se formaban, mediante un enfoque colaborativo y basado en tareas auténticas, para transferir los aprendizajes al diseño de sus propios cursos masivos (Osuna-Acedo, Marta-Lazo, y Frau-Meigs, 2018).

Todas estas iniciativas señalan la necesidad de influir en el diseño de los MOOC con la intención de garantizar su calidad (Conole, 2015; Littlejohn y Milligan, 2015). Conole (2015) propone doce dimensiones para evaluar y clasificar la calidad de los MOOC en función de su diseño: el grado de apertura, el alcance de la participación (o nivel de masificación), el nivel de uso de recursos multimedia, el nivel de comunicación, el grado de colaboración que se propone, el tipo de itinerario de aprendizaje que se propone a los aprendices (desde centrado en el aprendiz hasta altamente estructurado por el profesor), el sistema de garantía de calidad esperado, el grado en que se fomenta la reflexión del alumno, el tipo de certificación ofrecida, el nivel de formalidadinformalidad, la autonomía y la diversidad.

Uno de los aspectos críticos que aparece en diferentes investigaciones es el volumen de información que circula en un MOOC, que puede ser fácilmente desorientador y abrumador, especialmente para aquellos estudiantes con expectativas de instrucción similares a las de los modelos tradicionales de educación superior. Lograr una 
organización efectiva del aprendizaje y la gestión de recursos requiere de una gran autonomía y capacidad de autoorganización (McAuley, Stewart, Siemens, y Cormier, 2010; Tschofen y Mackness, 2012). Kop y Fournier (2011) analizaron la agencia y el nivel de autonomía requerido por los participantes en un MOOC, utilizando el modelo cuatridimensional de Bouchard sobre el control de los aprendices (Bouchard, 2009). Este estudio apunta a aspectos como la gestión del tiempo y de la información, la formulación de objetivos y la planificación efectiva, todos ellos directamente relacionados con la autorregulación del aprendizaje, como factores que influyen directamente en la participación y el tipo de actividades que los aprendices desarrollan en los MOOC.

Es así como el concepto de autorregulación (AR) del aprendizaje comienza a ser de interés para estudios sobre los MOOC (Pérez-Álvarez, Maldonado-Mahauad, y PérezSanagustín, 2018). La AR se define como "el conjunto de pensamientos, emociones y acciones planificadas y adaptadas cíclicamente para el logro de objetivos personales" (Zimmerman, 2000, p. 14). El modelo de AR propuesto por Zimmerman consta de tres fases (previsión, desarrollo y autorreflexión), cruzadas por subprocesos afectivos, conductuales, cognitivos y metacognitivos (entre ellos, la formulación de objetivos, la gestión del tiempo, la motivación, el autocontrol, la reflexión y autoevaluación, la percepción de autoeficacia y la autosatisfacción) (Fontana, Milligan, Littlejohn, y Margaryan, 2015; Zimmerman, 2000). Este enfoque pone el énfasis en la perspectiva de las diferencias individuales, aunque el contexto social se reconoce también como un componente del proceso de AR (Schunk y Zimmerman, 1997).

Uno de los trabajos de referencia en este respecto es el presentado en Littlejohn, Hood, Milligan, y Mustain (2016); Littlejohn y Milligan (2015); Margaryan et al. (2015); Milligan y Littlejohn (2014). Los autores analizan las estrategias de AR que los aprendices ponen en juego en los MOOC y cómo estas varían según diferentes factores relacionados con los aspectos motivacionales, como por ejemplo la percepción de los participantes acerca del propósito de este tipo de curso. Los resultados muestran que los aprendices con puntuaciones más altas en el aprendizaje autorregulado consideran el MOOC principalmente como una oportunidad para el desarrollo profesional y, por lo tanto, directamente relacionado con sus tareas, necesidades y roles en el lugar de trabajo.

Otra línea de investigación más reciente en el estudio de la regulación del aprendizaje desde el enfoque sociocultural se ha centrado en su dimensión social, dando lugar a nuevas construcciones a partir de las cuales analizar el proceso de regulación, como la corregulación (CR) y la regulación social compartida (RSC) (Hadwin, Järvelä, y Miller, 2011). El aprendizaje generalmente no es solo individual o colaborativo, por lo que entendemos que su estudio debe abordarse desde una perspectiva más holística, que tenga en cuenta el componente individual y social en diversas situaciones de aprendizaje. Esto tiene un sentido especial en entornos como los MOOC, en la mayoría de los cuales: a) se presupone la capacidad de AR del participante en el curso, que no necesariamente es real; b) el aprendizaje podría facilitarse mediante la CR de lo que se comparte, entendido como retroalimentación mutua, que no siempre funciona de manera efectiva, y c) se tiende a idealizar la generación espontánea de comunidades de aprendizaje que se regulan colaborativamente mediante su propia adquisición de conocimiento compartido.

Como apuntan Littlejohn y Milligan (2015) la mayor parte de los MOOC no presentan un diseño que promueva o facilite el aprendizaje autodirigido y autorregulado. Parece por lo tanto necesario lograr una mayor comprensión de los procesos de autodirección y de regulación del aprendizaje y desarrollar modelos y sistemas de apoyo para los aprendices en este tipo de contextos (Bonk, Lee, Kou, Xu, y Sheu, 2015). Para ello, es necesario examinar en profundidad los modelos pedagógicos que sustentan los MOOC, prestando especial atención a las experiencias y motivaciones de los alumnos, así como al valor que atribuyen a su participación en un entorno de aprendizaje abierto 
y en red (McAuley et al., 2010).

El trabajo presentado en este artículo se orienta por las siguientes preguntas de investigación:

- RQ1. ¿Qué aspectos deberían considerarse en el diseño de un sistema para apoyar la regulación social del aprendizaje en xMOOC?

- RQ2. ¿Cómo puede integrarse en el diseño de un xMOOC un sistema de apoyo a la regulación social del aprendizaje?

Con el fin de responder las preguntas planteadas, se realizó una revisión de literatura sobre el análisis de los tres procesos de regulación del aprendizaje mencionados (AR, CR y RSC) y sobre el uso de herramientas de andamiaje para apoyarlos en diferentes entornos. Esta revisión nos proporcionó el marco conceptual y los elementos de diseño que considerar en la construcción de un primer prototipo de sistema de apoyo a la regulación del aprendizaje para los MOOC.

Seguidamente, presentamos brevemente la base conceptual que articula nuestra propuesta. Posteriormente describimos los distintos elementos que constituyen el sistema de apoyo a la regulación y la forma en que estos interactúan entre sí. Esta parte del trabajo pretende responder a la primera pregunta de investigación planteada. Para poder trabajar en la segunda pregunta de investigación, propusimos un caso de intervención, basado en un XMOOC, donde integramos e implementamos el sistema de apoyo a la regulación diseñado. A continuación, se describe el caso de intervención y la integración del sistema de apoyo a la regulación en la plataforma del xMOOC, así como el diseño de la investigación y los instrumentos de recogida de datos que se utilizaron. Para terminar, se presentan algunos resultados de la implementación, que conducen a unas conclusiones finales.

\section{REVISIÓN DE LITERATURA}

\subsection{La dimension social de la regulación del aprendizaje}

El concepto de CR deriva de la consideración vygotskiana del aprendizaje, según la cual los procesos cognitivos superiores se internalizan a través de la interacción social. Desde esta perspectiva, la interacción social emergente con otros sujetos en un contexto dado da lugar a la internalización de los procesos de AR (McCaslin y Good, 1996; McCaslin y Hickey, 2001). Hadwin et al. (2011) definen la CR como una coordinación temporal de la AR del aprendizaje con otros, entendiendo que estos "otros" pueden ser los iguales o pares, el docente, etc. En los procesos de interacción social, la CR pone en juego procesos de negociación que hacen surgir la experiencia y las dificultades de AR de cada participante, mejorando así el logro de niveles más altos de regulación por parte de cada individuo. El estudio de los procesos de CR se basa en el análisis de las interacciones o dinámicas entre los sujetos, con respecto o al servicio de los procesos de regulación del aprendizaje.

Por otro lado, la regulación social compartida (RSC) del aprendizaje tiene lugar en contextos de colaboración y cooperación, en los que un grupo de individuos comparten sus procesos de regulación del aprendizaje (incluidas creencias, conocimientos, estrategias, etc.), de manera orquestada al servicio de un objetivo o producto común o co-construido (Hadwin et al., 2011; Panadero y Järvelä, 2015). La investigación sobre la RSC, por lo tanto, se centra en estos procesos de regulación compartidos, codependientes y co-construidos que se dan en interacción con el yo individual, pudiendo adoptar la forma de planificación compartida, objetivos compartidos, monitorización y evaluación compartidos, estrategias compartidas, etc. Su estudio se ha concentrado especialmente en el campo de los entornos CSCL (Computer Supported Collaborative 
Learning), en el análisis de los procesos de regulación de equipos durante el trabajo colaborativo.

En una extensa revisión del tema, Panadero y Järvelä (2015) enumeran varias líneas de investigación pendientes de abordar sobre la RSC, entre las cuales destacamos dos: a) la implementación de intervenciones para promover o apoyar este tipo de procesos, especialmente con ayuda de las tecnologías digitales y b) el estudio de estos procesos en interacción con los de AR y CR, ya que pueden considerarse interdependientes.

Autores como Hadwin et al. (2011) o Volet, Summers, y Thurman (2009) sostienen que, con el tipo correcto de tareas y condiciones sociales, estas tres formas de regulación del aprendizaje pueden manifestarse y ser estudiadas simultáneamente. Sin embargo, no es fácil encontrar estudios que aborden estos tres procesos en su conjunto y mucho menos en contextos de aprendizaje abierto, en los que la colaboración se articula de manera distribuida y emergente, sin responder claramente a una estructura de organización grupal formal dada. Precisamente por esta razón, parece esencial abordar esta brecha, con el propósito de identificar cómo estos tres procesos se manifiestan en contextos como los MOOC y cómo se pueden apoyar o facilitar a través de su diseño.

Nos interesa especialmente estudiar cómo se puede proporcionar un apoyo social en los procesos de regulación del aprendizaje en el marco de los XMOOC. Esto incluye procesos de modelado y andamiaje (McCaslin y Hickey, 2001), entre otros que puedan identificarse, ya sean proporcionados por los compañeros del curso, por el dinamizador, o bien mediados por el entorno mismo, como parte del diseño del curso.

\subsection{El uso de prompts como herramientas de andamiaje de los procesos de regulación del aprendizaje}

En los últimos años se han desarrollado algunas herramientas tecnológicas para apoyar los procesos de AR en MOOC. De acuerdo con la revisión de literatura elaborada por Pérez-Álvarez et al. (2018), estas herramientas utilizan diferentes mecanismos para apoyar la AR, como la visualización, la comparación social, la recomendación, la colaboración y las interfaces para la entrada de datos. Sin embargo, según refieren los autores, la mayoría de estas herramientas no se han evaluado en términos de impacto en el desarrollo de estrategias de AR por parte de los participantes. Es decir, en la mayoría de estas investigaciones no se ha previsto en el diseño qué indicadores permitirán establecer una relación entre las estrategias de AR apoyadas por las herramientas desarrolladas y la actividad de los participantes. Los mismos autores desarrollan la herramienta NoteMyProgress con esta finalidad (Pérez-Álvarez, Maldonado-Mahauad, Sapunar-Opazo, y Pérez-Sanagustín, 2017). Algunas de sus funcionalidades mejor valoradas son la posibilidad de tomar notas durante el estudio de los contenidos para utilizarlas más tarde, en términos de ayuda a la organización mental y la información sobre el tiempo dedicado a las actividades propuestas en el curso, o incluso el dedicado a otras actividades no directamente relacionadas con la actividad en el curso (procrastinación). Todo ello se valora como útil para la toma de conciencia y reconsideración de la inversión de tiempo.

El uso de herramientas para andamiar la regulación del aprendizaje y más específicamente el uso de prompts (avisos ${ }^{1}$ ) ha sido estudiado durante las últimas dos décadas (Bannert, 2009; Bannert y Reimann, 2012; Wirth, 2009). Según Bannert y Reimann (2012, p. 195), los prompts centran la atención de los alumnos en comprender las actividades de aprendizaje en las que participan. Pueden ser útiles para apoyar el recuerdo y la ejecución de conocimientos y habilidades durante el aprendizaje, como actividades, procedimientos, técnicas, o incluso el uso de estrategias cognitivas y metacognitivas.

\footnotetext{
${ }^{1}$ Mantenemos en el artículo el uso del término prompt en inglés, por los matices que aporta más allá del significado literal de "aviso" (potenciar, impulsar, procurar, recordar).
} 
Los prompts educativos toman la forma de intervenciones breves, integradas en un contexto de aprendizaje dado, como declaraciones explícitas que los estudiantes deben considerar durante su aprendizaje. Su objetivo es centrar la atención del alumno en aspectos específicos del proceso. En el caso concreto de la regulación del aprendizaje, el uso de prompts ha consistido principalmente en pedir a los estudiantes que realicen actividades de AR específicas, instándoles explícitamente a reflexionar, supervisar y revisar su proceso de aprendizaje. El supuesto de partida es que los prompts les permitirán activar su repertorio de conocimientos y estrategias metacognitivas durante el aprendizaje porque ya los poseen, aunque es posible que no los recuerden o no los ejecuten espontáneamente.

Las herramientas y los andamios de regulación también se han utilizado para promover la RSC en contextos de aprendizaje colaborativo apoyado por ordenador (CSCL). En este caso, el apoyo ha consistido en proponer a los estudiantes que negocien y compartan sus objetivos, planes y estrategias, así como que reflexionen sobre el logro de sus objetivos o evalúen sus planes y estrategias (Malmberg, Järvelä, Järvenoja, y Panadero, 2015). Järvelä et al. (2016, p. 267) mencionan tres principios de diseño para apoyar la RSC: 1) aumentar la conciencia de los estudiantes sobre el proceso de aprendizaje propio y ajeno, 2) apoyar la externalización del proceso de aprendizaje de los estudiantes en un plano social, ayudándoles a compartir y a interactuar entre sí, y 3) impulsar la adquisición y activación de procesos de regulación. Las herramientas de regulación podrían considerarse herramientas de sensibilización o toma de conciencia (awareness), ya que ayudan a hacer visibles los objetivos de AR de los distintos miembros del grupo y de esta manera aumentan las posibilidades de desarrollar estrategias de regulación socialmente compartidas (Järvelä et al., 2016, 2015).

Otro aspecto interesante de los prompts, como señalan Molenaar y Järvelä (2014), es que permiten recopilar datos sobre procesos o sucesos que se dan "sobre la marcha" (on-the-fly), que no podrían estar disponibles por otros medios.

\section{PROPUESTA DE DISEÑO DE UN SISTEMA DE APOYO A LA REGULACIÓN DE APRENDIZAJE PARA XMOOC}

El propósito del sistema de apoyo a la regulación del aprendizaje diseñado es doble: por una parte, tiene la intención de ayudar a los participantes a tener una experiencia de aprendizaje más satisfactoria, prevenir el desgaste y el abandono y explorar posibilidades para mejorar el diseño del xMOOC, prestando atención a las oportunidades de aprendizaje individuales y sociales. Por otra parte, pretende avanzar en la comprensión de la forma en que operan los procesos de regulación del aprendizaje (AR, CR y RSC) y cómo pueden recibir un mejor apoyo en entornos como los xMOOC. A continuación, describimos los elementos constitutivos del sistema de apoyo a la regulación diseñado:

\section{Tipos de prompts}

Los elementos básicos de andamiaje utilizados son los siguientes tres tipos de prompts:

- Prompts orientadores (GP): directrices que invitan a los participantes a realizar algunas acciones. Algunas de ellas tienen que ver con poner en práctica estrategias o procedimientos, otras con compartir y discutir las anteriores con el resto de los compañeros en el foro y por último, con reflexionar sobre el propio proceso de aprendizaje.

- Prompts reflexivos (RP): preguntas reflexivas planteadas a los participantes con el fin de estimular estrategias metacognitivas, donde se les pide dar respuesta por 
escrito sobre su propia experiencia de aprendizaje con respecto a: el uso de alguna estrategia o procedimiento, el intercambio y discusión con otros compañeros de curso y el proceso reflexivo mismo.

- Prompts de retroalimentación (FP): a nivel individual, consisten en el reenvío automático de las respuestas de cada participante a los prompts reflexivos; a nivel grupal, consisten en el reenvío de las respuestas de todos los miembros del grupo a las preguntas reflexivas sobre la actividad compartida.

Los prompts orientadores y los de retroalimentación tienen la intención de actuar principalmente como andamios del proceso de regulación, mientras que los prompts reflexivos, además de esto, nos permiten recopilar datos cualitativos del proceso "sobre la marcha", según tiene lugar la experiencia de los participantes a lo largo del desarrollo del MOOC.

\section{Fases de regulación}

En la estructuración del sistema de apoyo a la regulación, tomamos como referencia y adaptamos la propuesta de Bannert y Reimann (2012). Como en su caso, nuestra propuesta sigue las tres fases cíclicas de aprendizaje de Zimmerman (2000): previsión o planificación, desempeño y autorreflexión, con la diferencia de que nos referimos más directamente a los procesos relacionados con la estructura y el desarrollo de la tarea en cada fase. Además, subdividimos la fase de previsión en dos: una preliminar dedicada a la comprensión de la tarea y una posterior centrada en la planificación y el establecimiento de objetivos.

De esta manera, la provisión de los tres tipos de prompts se distribuye en las cuatro fases siguientes a lo largo del curso:

- Fase 1: Comprensión del reto de aprendizaje del MOOC.

- Fase 2: Establecimiento de objetivos de aprendizaje y planificación

- Fase 3: Desarrollo de la actividad

- Fase 4: Evaluación del desempeño y adaptación

Para la formulación específica de los prompts de cada fase, nos basamos por una parte en la propuesta de Bannert y Reimann (2012), especialmente en lo que se refiere a los procesos de AR, pero la adaptamos tomando como referencia otras propuestas para apoyar la CR (Kaplan, Montalembert, Laurent, y Fenouillet, 2017; Volet et al., 2009) y la RSC (Järvelä et al., 2016; Morris et al., 2010).

\section{Objetivos de regulación}

Nuestro propósito es considerar las diferentes dimensiones que se superponen en la regulación del proceso de aprendizaje: cognitiva, conductual, motivacional y socioemocional. Esto significa que los prompts tienen la intención de activar en los participantes las distintas dimensiones u objetivos de regulación. Por otra parte, además de la actividad metacognitiva que tendría un papel transversal, el objetivo es que los prompts induzcan los procesos de regulación centrales en las distintas fases del proceso, es decir, los de orientación, planificación, formulación de objetivos, búsqueda de información relevante, monitorización y evaluación.

\section{Naturaleza de la tarea}

Otro aspecto que debe tenerse en cuenta es la naturaleza de la tarea considerada en este caso. Distinguimos entre dos tipos básicos de actividades de aprendizaje que los participantes deben desarrollar en el MOOC: 
- Actividad individual: se refiere a la actividad general en el MOOC que consiste en trabajar con la documentación y los materiales proporcionados y responder al cuestionario final de cada módulo. También implica la interacción social no estructurada y el intercambio con otros participantes en el espacio del foro.

- Actividad colaborativa: se refiere a actividades específicas que los participantes deben llevar a cabo en grupo e implican negociación e intercambio con sus miembros. Específicamente para este caso, se trató de una actividad opcional de revisión individual por pares que fue rediseñada como actividad colaborativa, de modo que implicara compartición de conocimientos, negociación de objetivos y procedimientos, planificación y revision del proceso conjunta, etc. de forma que pudiera conducir a procesos de RSC.

El hecho de diferenciar entre la actividad individual y la colaborativa en el MOOC nos permitió diseñar y administrar prompts apropiados para cada caso, con el fin de estimular los procesos de regulación correspondientes: para la actividad individual los relacionados con la AR y la CR y para la actividad colaborativa, los dos anteriores, además de los referidos a la RSC.

Para evitar sobrecargar a los estudiantes, tratamos de simplificar la administración de prompts al máximo. Así, en el caso de la actividad individual, para estructurar la presentación de los prompts, decidimos considerar todo el curso como una sola tarea y de este modo, distribuir las cuatro fases de regulación a lo largo de los 4+1 módulos del MOOC, es decir, durante las cuatro semanas y media de duración.

La actividad de colaboración se propuso en el tercer módulo del MOOC y se consideró globalmente como una tarea, de una semana de duración. En este caso, teniendo en cuenta el breve periodo de tiempo con que se contaba para el desarrollo de la actividad, las fases de regulación y la provisión de los prompts se simplificaron aún más, considerando tres fases o momentos de regulación a lo largo de la semana, de modo que no resultara tan intensivo para los participantes.

\section{CASO DE INTERVENCIÓN: UN XMOOC SOBRE GAMIFICACIÓN}

\subsection{Metodología e instrumentación}

Se utiliza un modelo de investigación basado en el diseño, mediante el cual la investigación se propone generar y analizar cambios en la práctica de la regulación del aprendizaje en los MOOC, a partir del desarrollo e implementación de una solución de diseño. En paralelo, se pretende avanzar en el desarrollo de teoría sobre el apoyo de los procesos de regulación del aprendizaje basado en el uso de prompts en este tipo de contextos. Con este doble propósito, la investigación sigue un ciclo continuo de diseño, intervención, análisis, evaluación, reflexión y rediseño.

Para la recogida, análisis e interpretación de los datos, se utiliza un planteamiento mixto, aunque con preeminencia del enfoque cualitativo. Se recogen datos cuantitativos de las estadísticas de participación generadas por la plataforma MiriadaX, pero la mayor parte de fuentes de información son de tipo cualitativo: entrevistas semiestructuradas con participantes del MOOC, respuestas obtenidas en los prompts reflexivos en las distintas fases de aplicación del sistema de apoyo y, finalmente, los registros de la interacción entre los participantes en la plataforma (foro, wiki y blog). También se dispone de los datos de la encuesta de satisfacción general de los participantes con el MOOC. La recogida de datos se realiza en cada uno de los módulos a través de los instrumentos mencionados, excepto en el caso de las entrevistas y la encuesta que se realizan al finalizar el MOOC. 


\subsection{Escenario de investigación y participantes}

El curso "Introducción a la gamificación a través de casos prácticos" se utiliza como caso para diseñar e implementar el sistema de apoyo a la regulación. Se trata de un xMOOC prototípico. Está estructurado en cuatro módulos además del Módulo 0 de tipo introductorio y su duración es de cuatro semanas y media. Los dos primeros módulos están dedicados a proporcionar conocimientos básicos sobre los juegos y los elementos de gamificación, así como pautas para el diseño de soluciones de aprendizaje gamificadas, mientras que el tercer y cuarto módulo se centran en casos prácticos en el campo de la salud y la educación respectivamente. La estructura de cada módulo es la habitual en un xMOOC: los contenidos se presentan a través de texto y videos; hay una prueba de respuesta múltiple al final de cada modulo como principal herramienta de evaluación de la adquisición de conocimientos de los participantes en el curso. Esta prueba proporciona a los participantes una nota y aprobarla es el único requisito para superar el módulo y el curso en su conjunto. El tercer y cuarto módulos también incluyen tareas opcionales que implican un poco más de desarrollo escrito. Estas actividades son evaluadas por los propios participantes a través de un procedimiento de evaluación por pares facilitado por la plataforma de aprendizaje. Deben completarse en un período de tiempo específico para ser tomadas en cuenta, aunque no se consideran en la calificación final del curso. El MOOC también presenta casos prácticos de éxito y propone el uso de una herramienta de gestión de tareas gamificada llamada Habítica. Así mismo, se invita a los participantes a participar en el espacio de foro que ofrece la plataforma, pero no existen actividades obligatorias que impliquen su uso para discutir o colaborar. El MOOC está alojado en la plataforma de aprendizaje MiriadaX, una iniciativa de Telefónica Educación Digital que opera desde 2013, con especial incidencia en el territorio de América Latina.

Los participantes potenciales en la investigación son todos los inscritos en la $3^{\mathrm{a}}$ edición del MOOC (marzo-abril), un total de 3.177. De este total, se registran voluntariamente para seguir el sistema de apoyo a la regulación implementado 234 participantes, de los cuales 104 a la actividad individual, 13 a la colaborativa y 118 a ambas. Si atendemos a las respuestas a los prompts reflexivos planteados en cada módulo, podemos decir que el porcentaje va decreciendo progresivamente de forma similar a la participación general en el MOOC. La actividad colaborativa propuesta no es desarrollada por ningún grupo finalmente. Las razones expresadas por los participantes tienen que ver con la falta de tiempo y también con trabajos de mantenimiento y actualización tecnológicos de la plataforma que, por una parte provocan problemas técnicos que impiden el acceso al MOOC, y después originaron cambios en el acceso a las herramientas necesarias para la realización de la actividad, precisamente el dia en que se planteaba su inicio.

\subsection{Implementación del sistema de regulación en MiriadaX}

La integración de la capa de diseño en el xMOOC se realizó principalmente aprovechando las funcionalidades y herramientas proporcionadas por la plataforma MiriadaX. Prevaleció el criterio de evitar agregar complejidad al entorno de aprendizaje. Solo para el caso de los prompts reflexivos, se utilizaron enlaces a formularios básicos externos de Google (Google Forms).

En el Módulo 0 se presentaba el sistema de apoyo a la regulación bajo el epígrafe: "Dirije y mejora tu aprendizaje". En esta presentación, se invitaba a los participantes a usar el sistema propuesto para ayudarles a dirigir y mejorar su experiencia de aprendizaje. De forma voluntaria, podrían registrarse a través de un formulario para realizar la actividad individual, la actividad colaborativa o ambas. Esto nos permitiría tener cierto control sobre los participantes inscritos inicialmente y hacer seguimiento de su continuidad. 
A continuación, creamos un itinerario para la actividad individual y otro para la actividad de colaboración y en cada caso incorporamos el sistema de apoyo a la regulación en el MOOC, según se describe a continuación:

A) Para la actividad individual:

- Introducción: se realiza una breve introducción de cada fase de regulación al final del módulo en que cada una de ellas se inserta.

- Presentación de prompts orientadores: en el blog de la plataforma se presentan, para cada una de las fases de apoyo a la regulación, los prompts orientadores correspondientes.

- Interacción social: en cada fase algunos prompts orientadores sugieren a los participantes compartir y discutir sus estrategias, reflexiones, opiniones y sensaciones en el foro durante todo el proceso. Por lo tanto, el foro se propone como el espacio principal para la interacción y la discusión social abierta. Los participantes también pueden hacer comentarios a las entradas del blog en que se van presentando las fases del sistema de apoyo a la regulación.

- Presentación de prompts reflexivos: se administran a través de enlaces a formularios de Google dentro de cada una de las publicaciones del blog.

- Presentación de prompts de retroalimentación: son reenviados a cada participante de forma automática cada vez que este responde a un formulario de prompts reflexivos.

B) Para la actividad colaborativa:

- Introducción: se realiza una breve presentación de la actividad colaborativa en la descripción del tercer módulo. En esta presentación se deja claro que esta actividad de revisión por pares se puede realizar de manera individual o colaborativa, siempre de forma opcional.

- Descripción de la actividad colaborativa: la descripción general se realiza en el blog de la plataforma.

- Presentación de prompts orientadores: se presentan en el espacio wiki de la plataforma a través de una propuesta simplificada en tres fases (en lugar de cuatro) de apoyo a la regulación. Es decir, en el mismo espacio propuesto para la realización grupal de la actividad colaborativa. Cada grupo dispone de una página wiki para resolver la actividad. Cada página wiki incluye una plantilla para facilitar la estructura del trabajo y el desarrollo de la actividad colaborativa. La conformación de grupos, de un máximo de tres miembros, se deja en manos de los participantes y se propone que anoten sus nombres al inicio de la página wiki donde realizarán la actividad.

- Interacción social: el espacio de foro se propone adicionalmente, complementando al wiki, como lugar de discusión, negociación y toma de decisiones del grupo.

- Presentación de prompts reflexivos: se administran a través de enlaces a formularios de Google desde la misma página wiki.

- Presentación de prompts de retroalimentación: la intención es compartir manualmente con cada grupo las respuestas a los prompts reflexivos del resto de miembros.

Dado que el sistema de apoyo a la regulación se distribuyó en diferentes espacios de la plataforma, vinculamos estos espacios entre sí, de la manera más clara posible, mediante la explicación del sistema y el uso de hipervínculos. La finalidad era hacer 
explícita y comprensible la estructura y lógica interna del sistema y a la vez facilitar su seguimiento dentro y a lo largo del desarrollo del MOOC.

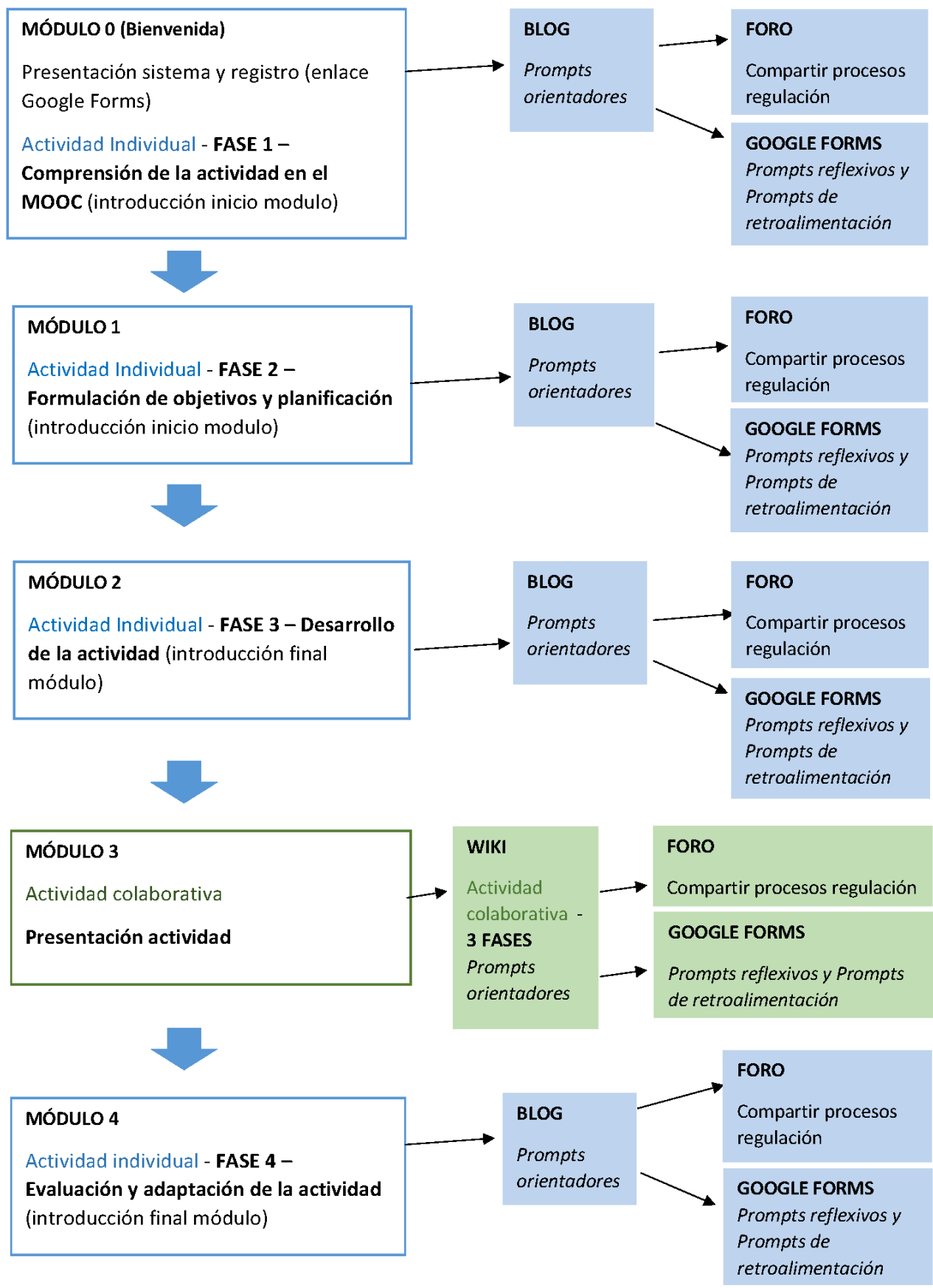

Figura 1. Mapa de integración del sistema en la plataforma MiríadaX. Elaboración propia.

\section{ANÁLISIS Y RESULTADOS DE LAS ENTREVISTAS}

A continuación, se presentan los resultados de las entrevistas semiestructuradas realizadas a cuatro participantes. Todos ellos se inscribieron en el sistema de apoyo a la regulación, tres de ellos a la parte de actividad invidual y uno a la individual y también a la colaborativa. Solo uno de ellos realizó un seguimiento completo del sistema, 
los otros tres realizaron un seguimiento parcial. Todos ellos completaron el MOOC prácticamente en su totalidad, dos de ellos realizando también las actividades $\mathrm{P} 2 \mathrm{P}$ en su version individual. Ninguno de ellos desarrolló finalmente la parte de actividad colaborativa propuesta por el sistema.

El guión de entrevista se estructuró en diversos subapartados que agrupaban preguntas referidas a los siguientes aspectos: a) Perfil del participante y contexto; b) Aspectos motivacionales; c) Comprensión del sistema; d) Utilidad del sistema; e) Aplicación de estrategias de regulación; f) Valoración del diseño del sistema.

Las entrevistas, de unos 30 minutos de duración, fueron transcritas y codificadas partiendo de un conjunto de categorías preestablecidas siguiendo los temas incluidos en la estructura del guión de entrevista. Estas categorías fueron desgranándose y redefiniéndose en el mismo proceso de codificación con el propósito de identificar aspectos emergentes y acercar al máximo el marco de análisis utilizado a los datos recogidos. Seguidamente presentamos los resultados obtenidos en las entrevistas organizados a partir de las preguntas de investigación planteadas.

\section{RQ1: Utilidad y uso del sistema}

Dentro de la valoración de la utilidad del sistema desde el punto de vista del apoyo a la regulación por parte de los participantes contemplamos las distintas dimensiones de la regulación del aprendizaje en que el sistema pretende influir: aspectos motivacionales, aspectos cognitivos y conductuales, aspectos metacognitivos y aspectos relacionales o sociales.

\section{- Aspectos motivacionales:}

En cuanto a los aspectos motivacionales distinguimos entre los motivos que llevaron a los participantes a utilizar el sistema y su posicionamiento y expectativas generales respecto al desarrollo del curso. En cuanto a lo primero, la razón más clara fue el hecho de que pudiera actuar como punto de anclaje o aliciente para continuar en el MOOC hasta su finalización. También se apuntan aspectos como "la curiosidad" o "ver en qué consistía el sistema". El hecho de que el seguimiento del sistema se plantease como algo opcional, que no implicaría gran esfuerzo o dedicación, fueron otros factores que influyeron en la decisión.

En general, los participantes tienen un posicionamiento concreto desde el inicio del curso respecto al nivel de implicación que van a tener y, en consecuencia, respecto al tipo de dedicación y la metodología de trabajo que van a seguir. Por ese motivo, tres de los entrevistados decidieron no inscribirse en la actividad colaborativa, ya que tenían claro que no podrían cumplir con el compromiso que requería. En el caso de la participante que sí se apuntó, reconoce que no se fijó demasiado ni pensó en lo que podría implicar. Simplemente, en el momento de inscribirse, valoró positivamente la posibilidad de intercambio con otros participantes de intereses similares.

\section{- Aspectos cognitivos y conductuales:}

Los entrevistados valoran la utilidad del sistema en el apoyo de estrategias de planificación. De todas formas, en su mayoría reconocen que el sistema no cambió sus estrategias ni su enfoque de aprendizaje en el curso. Tenían claro que su intención no era profundizar al máximo en los contenidos y propuestas del MOOC, ya fuera por una cuestión de falta de tiempo, o por el hecho de tratarse de una actividad complementaria sobre una temática de la que ya tenían algún conocimiento previo. Reconocen que si hubieran tenido interés en profundizar más podrían haber sacado más partido del sistema, pero que optaron por no hacerlo. En general, la estrategia de seguimiento del MOOC por parte de los participantes consistía en entrar en algún momento de la semana y avanzar todo lo posible, es decir, hasta cerrar un módulo o una temática. Por 
lo tanto, predomina una estrategia intensiva e intermitente sobre una más progresiva o dosificada y constante.

El MOOC proponía el uso de una herramienta de gamificación llamada Habítica aplicada a la gestión de tareas dentro del propio curso. A pesar de no haberla contemplado como parte del sistema de apoyo a la regulación, vemos que para alguno de los participantes funcionó como herramienta de apoyo en distintos sentidos (motivacional, estratégico, cognitivo) por lo tanto, parece interesante considerar las posibilidades que ésta ofrece para complementar el sistema propuesto en próximas implementaciones.

Otras estrategias de aprendizaje empleadas por los participantes, por ejemplo, en caso de no comprender bien los contenidos, son: visualizar varias veces los vídeos, consultar las lecturas complementarias sugeridas en el módulo, realizar búsquedas en Internet, etc. En general, no se planteaban realizar consultas en el foro por motivos que abordamos en el apartado de aspectos relacionales. Una de las participantes utilizaba también la estrategia de tomar notas o apuntes para recordar mejor los contenidos de los temas a la hora de realizar los tests de evaluación de cada módulo.

\section{- Aspectos metacognitivos:}

En cuanto a los procesos metacognitivos que se pretendían incentivar con el sistema, especialmente mediante los prompts reflexivos y los prompts de retroalimentación, vemos que la valoración es diversa. Mientras que para dos de los entrevistados resultaron útiles para "mirar atras" y valorar si se estaba cumpliendo con lo planificado, o cómo se estaba trabajando y si las estrategias y procedimientos en marcha daban buen resultado, para otros no funcionaron. En algún caso, se reconoce que ya de entrada no interesaba profundizar en el proceso de aprendizaje ni modificar las estrategias empleadas. En otro, resultaba poco motivador el formato de cuestionario y el hecho de que al otro lado no hubiera nadie para recibir la respuesta.

Por el mismo motivo, en dos casos los participantes valoraron como no necesarios los prompts de retroalimentación. A pesar de ello, reconocieron que sí podría haber resultado útil recuperar las propias respuestas en caso de haberse propuesto reflexionar sobre el propio proceso de aprendizaje a lo largo del curso, para facilitar el seguimiento de los cambios de planteamiento, estrategia utilizada, etc. durante el proceso.

\section{- Aspectos relacionales / sociales:}

La dimension social hace referencia fundamentalmente a los intercambios, comentarios y aportaciones realizados en el espacio de foro. A pesar de no haber utilizado el foro con la frecuencia y finalidad que proponían los prompts orientadores del sistema, sí parece que estos han servido para que los participantes se hicieran más conscientes del papel que puede jugar este espacio como punto de encuentro para intercambiar, no solo conocimientos, sinó también estrategias de aprendizaje concretas y la propia experiencia de realización del MOOC. De hecho, algún participante da a entender que, el hecho de compartir con el resto las propias reflexiones y actuaciones en el foro contribuye a darles sentido y por lo tanto puede incentivarlas.

A pesar de todo y excepto en casos puntuales, las consignas concretas del sistema relacionadas con la participación en el foro se siguieron muy poco. El argumento más generalizado de los entrevistados es que, en general, el nivel de lo que se comparte en el foro es pobre, cuesta encontrar aportaciones relevantes entre tanto ruido y por lo tanto acaba perdiendo el interés.

La reducida y dispersa interacción social registrada en el foro hace que resulte muy difícil identificar de forma clara evidencias de procesos de CR y de RSC.

\section{RQ2: Integración del sistema en el MOOC}


Las respuestas de los entrevistados nos llevan a interpretar que, aunque sí se comprendió el propósito y formato de presentación general del sistema de apoyo a la regulación, no acabó de interiorizarse su estructura y lógica interna. Más bien, reconocen que, cuando encontraban la información relativa a un apartado del sistema de apoyo durante el desarrollo del curso, si les iba bien en ese momento completaban lo solicitado por los prompts, aunque fuera parcial o superficialmente. Es decir, no se propusieron completar todas las fases del sistema, ya que no era prescriptivo para superar el MOOC.

El hecho de que el sistema de provisión de prompts se encontrara distribuido en distintos espacios o funcionalidades del MOOC difuminó el itinerario que pretendía dibujar. A pesar de ello, uno de los participantes valora como aspecto positivo el hecho de que permitiera conocer más espacios de la plataforma y de este modo generara una sensación de más seguridad para moverse dentro del entorno.

Uno de los aspectos en que hay mayor acuerdo entre los entrevistados es en observar que el formato de los prompts reflexivos no era el más adecuado para incentivar la respuesta. En este sentido, algunas de las sugerencias de los participantes son: administrarlos de forma más paulatina (preguntas puntuales a lo largo de todo el módulo) en lugar de hacerlo en bloque al final o al principio del módulo, evocar respuestas concretas más que reflexiones amplias, hacer que tengan un sentido/efecto concreto dentro del curso (evitar el efecto de estar perdiendo el tiempo hablando con una máquina).

Diseño de los procesos colaborativos:

Otra de las cuestiones críticas en las que hay acuerdo entre los entrevistados es en la problemática que plantea la colaboración como metodología de aprendizaje dentro del MOOC. La falta de tiempo para organizarse, el pudor o la falta de seguridad en las capacidades de uno mismo, el hecho de no conocer al resto de participantes para formar grupo, la desconfianza respecto el nivel de compromiso e implicación de los otros, la dificultad de gestionar el proceso de conformación de grupo, etc. son algunos de los factores que se destacan como inhibidores.

En cuanto a las posibles sugerencias para viabilizar los procesos colaborativos en el marco de los MOOC, los participantes apuntan ideas diversas, en ocasiones contradictorias como: promover situaciones en que los participantes puedan conocerse antes de iniciar los procesos de colaboración, ya sea en forma de actividades en el foro, chats síncronos, o incluso encuentros presenciales; plantear la colaboración solo en tareas puntuales y concretas que puedan realizarse de manera asíncrona y partir de la realización de proyectos propios para el planteamiento de las actividades de colaboración, que permitan una aplicación real de lo aprendido, serían otras propuestas a este respecto.

\section{CONCLUSIONES Y RECOMENDACIONES}

La primera implementación exploratoria del sistema de apoyo a la regulación del aprendizaje nos permite extraer unas primeras reflexiones.

En cuanto a la pregunta de investigación: ¿Qué aspectos deberían considerarse en el diseño de un sistema para apoyar la regulación social del aprendizaje en xMOOC?

El uso de prompts parece apropiado para inducir y apoyar de manera sostenida los procesos de regulación. Sin embargo, el bajo seguimiento del sistema por parte de los participantes, así como las aportaciones de los entrevistados, nos llevan a pensar que la presentación de los prompts orientadores, así como de los prompts reflexivos, debe repensarse, en el sentido de simplificarse y hacerse más transparente para los participantes a lo largo del MOOC. En el caso de los prompts reflexivos parece que el 
"efecto cuestionario" ha hecho que no se contemple con una herramienta de autogestión del proceso. En este sentido, una posibilidad seria plantear este tipo de prompts más en la línea de una funcionalidad de tomar notas a modo de diario reflexivo, similar a la de la herramienta NoteMyProgress (Pérez-Álvarez et al., 2017). Otra posibilidad sería mantener los prompts reflexivos pero hacer públicas las respuestas para todos los participantes, de modo anónimo, de manera que todos pudieran beneficiarse de las respuestas de los demás. Esto podría disminuir la sensación de estar respondiendo a una máquina y de falta de utilidad de estas respuestas reportada por algunos participantes. Funcionaría, de hecho, com un nuevo tipo de prompts de retroalimentación. Otra posibilidad sería probar el uso de prompts dirigidos que proporcionen a los aprendices modelos expertos de pensamiento reflexivo (Ifenthaler, 2012) que puedan funcionar como referencias.

Un aspecto que claramente determina el uso del sistema es la forma en que los participantes se posicionan y enfocan el aprendizaje en el MOOC desde su inicio, que tiende a ser superficial, intermitente e intensiva. Es difícil que los procesos de regulación, en especial los de tipo metacognitivo, se activen dentro de un planteamiento tan fragmentado. En este sentido, el sistema de apoyo a la regulación debería ayudar a sostener un recorrido más contínuo, progresivo y de sentido dentro del MOOC.

Por otra parte, la actividad colaborativa debe explicitarse e incentivarse de forma más clara desde un inicio y tal vez no presentarse a los participantes como un itinerario distinto del individual, sinó como un elemento más del mismo itinerario, que como el resto puede realizarse opcionalmente. Para viabilizar su realización y a la vez hacer posible el seguimiento de las fases de apoyo a la regulación debe considerarse un período de tiempo más amplio. El hecho de que sea una actividad opcional en el tercer módulo reduce en gran medida la participación, que ya es de por si escasa en las actividades individuales P2P en este momento del calendario del MOOC. Algo similar ocurre con cualquier forma de interacción social en el foro, como pre-requisito para que se produzcan procesos de CR y RSC. Por lo tanto, es necesario profundizar en las razones de la baja participación y generar mecanismos en los primeros módulos para hacer que la interacción social y la colaboración en el MOOC resulten más atractivas, productivas y gratificantes.

En cuanto a la pregunta de investigación: ¿Cómo puede integrarse en el diseño de un XMOOC un sistema de apoyo a la regulación social del aprendizaje?

La forma en que el sistema de apoyo a la regulación se integra en el diseño del MOOC parece una cuestión esencial para lograr un aprovechamiento óptimo por parte de los participantes. Lo cierto es que las características y la forma en que opera MiriadaX no facilitan una integración orgánica y al mismo tiempo atractiva del sistema, ya que se trata de una plataforma poco fexible y con pocas herramientas y funcionalidades pensadas para facilitar la interacción social y la colaboración. Aunque se trató de trazar lo más claramente posible el itinerario que seguía el sistema de apoyo dentro del entorno xMOOC, la desintegración de los diferentes componentes hizo que los participantes se perdieran con frecuencia y no entendieran completamente la propuesta. En las entrevistas se detecta que a los participantes les cuesta situar con claridad los aspectos referidos al sistema de apoyo a la regulación. En este sentido, si bien consideramos que esta capa de apoyo debe resultar más transparente para los participantes, al mismo tiempo debe dibujar un camino que sea reconocible y comprensible. Debe permitir tomar conciencia del proceso de regulación del aprendizaje dentro del MOOC y del sentido de hacer un uso apropiado y continuado de los prompts (Bannert y Reimann, 2012). Esto implica buscar estrategias para integrar mejor el sistema en los contenidos y la actividad de aprendizaje del MOOC (contemplando lo objetivos del módulo, contenidos, actividades y herramientas). En concreto los prompts, en lugar de presentarse en bloque, pueden administrarse de manera más paulatina e integrada en los contenidos del MOOC. Se podría incluso tratar de darles un tratamiento gamificado 
aprovechando los contenidos del curso.

Tal como se ha observado en otros estudios, ofrecer prompts para mejorar la regulación del aprendizaje no tiene porqué resultar suficiente (Bannert y Reimann, 2012). En próximas iteraciones de la investigación pretendemos analizar por qué los aprendices utilizan o no de la manera y con la frecuencia prevista estos prompts. En este sentido puede ser interesante analizar hasta que punto estos estudiantes disponen o no de las habilidades de autoregulación que se está tratando de activar o incentivar. Otra posibilidad es que sean necesarias ciertas orientaciones adicionales para "entrenar" un uso adecuado del sistema.

Las conclusiones extraidas en la primera iteración de la investigación ofrecen algunas pautas sobre cómo plantear mecanismos de apoyo a los procesos de regulación en un sentido amplio, dentro de un planteamiento general del diseño del aprendizaje en los MOOC.

\section{REFERENCIAS}

Anderson, T., y McGreal, R. (2012). Disruptive Pedagogies and Technologies in Universities. Educational Technology \& Society, 15(4), 380-389. Descargado de http:// citeseerx.ist.psu.edu/viewdoc/download?doi=10.1.1.298.6543\&rep=rep1\&type=pdf

Bannert, M. (2009). Promoting self-regulated learning through prompts: A discussion. German fournal of Educational Psychology, 23, 139-145. https://doi.org/10.1024/1010-0652.23.2.139

Bannert, M., y Reimann, P. (2012). Supporting self-regulated hypermedia learning through prompts. Instructional Science, 40(1), 193-211. Descargado de https://www.jstor.org/stable/43575195 https://doi.org/10.1007/s11251-011-9167-4

Bonk, C. J., Lee, M. M., Kou, X., Xu, S., y Sheu, F. R. (2015). Understanding the Self-Directed Online Learning Preferences, Goals, Achievements, and Challenges of MIT OpenCourseWare Subscribers. Educational Technology \& Society, 18(2), 349-365.

Bouchard, P. (2009). Pedagogy without a teacher: What are the limits? International fournal of Self-Directed Learning, 6(2), 13-22.

Clarà, M., y Barbera, E. (2013). Learning online: massive open online courses (MOOCs), connectivism, and cultural psychology, 34, 129-136. https://doi.org/10.1080/01587919.2013.770428

Clarà, M., y Barberà, E. (2014). Three problems with the connectivist conception of learning. Journal of Computer Assisted Learning, 30(3), 197-206. https://doi.org/10.1111/jcal.12040

Conole, G. (2015). MOOCs as disruptive technologies: strategies for enhancing the learner experience and quality of MOOCs. Revista de Educación a Distancia, 39, 1-17. Descargado de https://revistas.um.es/red/article/view/234221

Daniel, J. (2012). Making Sense of MOOCs: Musings in a Maze of Myth, Paradox and Possibility. fournal of Interactive Media in Education, 2012(3). Art. 18.. http://doi.org/10.5334/2012-18

Fidalgo-Blanco, A., Sein-Echaluce, M. L., y García-Peñalvo, F. J. (2016). From massive access to cooperation: lessons learned and proven results of a hybrid xMOOC/ cMOOC pedagogical approach to MOOCs. International fournal of Educational Technology in Higher Education, 13(24), 1-13. https://doi.org/10.1186/s41239-016-0024-z

Fontana, R. P., Milligan, C., Littlejohn, A., y Margaryan, A. (2015). Measuring self-regulated learning in the workplace. International fournal of Training and Development, 19(1), 32-52. https://doi.org/10.1111/ijtd.12046

García-Peñalvo, F. J., Fidalgo-Blanco, A., y Sein-Echaluce, M. L. (2018). An adaptive hybrid MOOC model: Disrupting the MOOC concept in higher education. Telematics and Informatics, 35(4), 1018-1030. https://doi.org/10.1016/j.tele.2017.09.012 
Guàrdia, L., Maina, M., y Sangrà, A. (2013). MOOC Design Principles. A Pedagogical Approach from the Learner's Perspective. eLearning Papers, 33, 1-6. Descargado de http://hdl.handle.net/10609/41681

Hadwin, A., Järvelä, S., y Miller, M. (2011). Self-Regulated, Co-Regulated, and Socially Shared Regulation of Learning. En B. Zimmerman y D. Schunk (Eds.), Educational psychology handbook series. Handbook of self-regulation of learning and performance (pp. 65-84). Routledge/Taylor \& Francis Group.

Ifenthaler, D. (2012). Determining the effectiveness of prompts for self-regulated learning in problema-solving scenarios. Educational Technology \& Society, 15(1), 38-52.

Järvelä, S., Kirschner, P. A., Hadwin, A., Järvenoja, H., Malmberg, J., Miller, M., y Laru, J. (2016). Socially shared regulation of learning in CSCL: Understanding and prompting individual-and group-level shared regulatory activities. International fournal of Computer-Supported Collaborative Learning, 11, 263-280. Supported Collaborative Learning. https://doi.org/10.1007/s11412-016-9238-2

Järvelä, S., Kirschner, P. A., Panadero, E., Malmberg, J., Phielix, C., Jaspers, J., ... Järvenoja, H. (2015). Enhancing socially shared regulation in collaborative learning groups: designing for CSCL regulation tools. Educational Technology Research and Development, 63(1), 125-142. https://doi.org/10.1007/s11423-014-9358-1

Kaplan, J., Montalembert, M. D., Laurent, P., y Fenouillet, F. (2017). ERICA- an instrument to measure individual and collective regulation of learning. Revue Européenne de Psychologie Appliquée/European Review of Applied Psychology, 67(2), 79-89. https://doi.org/10.1016/j.erap.2017.01.001

Kop, R., y Fournier, H. (2011). New dimensions to self-directed learning in an open networked learning environment. International fournal of Self-Directed Learning, 7(2), 1-18. Descargado de https://nrc-publications.canada.ca/eng/view/accepted/ ?id=c4dc46c9-ef59-46b8-af01-4a7fec44b023

Littlejohn, A., Hood, N., Milligan, C., y Mustain, P. (2016). Learning in MOOCs: Motivations and self-regulated learning in MOOCs. Learning in MOOCs: Motivations and self-regulated learning in MOOCs. The Internet and Higher Education, 29, 40-48. https://doi.org/10.1016/j.iheduc.2015.12.003

Littlejohn, A., y Milligan, C. (2015). Designing MOOCs for professional learners: Tools and patterns to encourage self-regulated learning. eLearning Papers, 42, 1-10. Descargado de http://oro.open.ac.uk/id/eprint/46385

Malmberg, J., Järvelä, S., Järvenoja, H., y Panadero, E. (2015). Promoting socially shared regulation of learning in CSCL: Progress of socially shared regulation among high-and low-performing groups. Computers in Human Behavior, 52, 562-572.

https://doi.org/10.1016/j.chb.2015.03.082

Margaryan, A., Bianco, M., y Littlejohn, A. (2015). Instructional quality of Massive Open Online Courses (MOOCs). Computers \& Education, 80, 77-83.

https://doi.org/10.1016/j.compedu.2014.08.005

McAuley, A., Stewart, B., Siemens, G., y Cormier, D. (2010). The MOOC model for digital practice. Charlottetown, Canada: University of Price Edward Island. Descargado de https://www.oerknowledgecloud.org/archive/MOOC_Final.pdf

McCaslin, M., y Good, T. L. (1996). The informal curriculum. En B. Berliner y R. Calfee (Eds.), Handbook of educational psychology (pp. 622-670). Macmillan Library Reference Usa/ Prentice Hall International.

McCaslin, M., y Hickey, D. T. (2001). Educational psychology, social constructivism, and educational practice: A case of emergent identity. Educational Psychologist, 36(2), 133-140. https://doi.org/10.1207/S15326985EP3602_8

Milligan, C., y Littlejohn, A. (2014). Supporting professional learning in a massive open online course. The International Review of Research in Open and Distance Learning, 15(5), 197-213. https://doi.org/10.19173/irrodl.v15i5.1855

Molenaar, I., y Järvelä, S. (2014). Sequential and temporal characteristics of self and socially 
regulated learning. Metacognition and Learning, 9, 75-85.

https://doi.org/10.1007/s11409-014-9114-2

Morris, R., Hadwin, A. F., Gress, C. L., Miller, M., Fior, M., Church, H., y Winne, P. H. (2010). Designing roles, scripts, and prompts to support CSCL in gStudy. Computers in Human Behavior, 26(5), 815-824. https://doi.org/10.1016/j.chb.2008.12.001

Osuna-Acedo, S., Marta-Lazo, C., y Frau-Meigs, D. (2018). De sMOOC a tMOOC, el aprendizaje hacia la transferencia profesional: El proyecto europeo ECO. Comunicar: Revista científica iberoamericana de comunicación y educación, 55, 105-114. https://doi.org/10.3916/C55-2018-10

Panadero, E., y Järvelä, S. (2015). Socially shared regulation of learning: A review. European Psychologist, 20(3), 190-203. https://doi.org/10.1027/1016-9040/a000226

Pérez-Álvarez, R., Maldonado-Mahauad, J., y Pérez-Sanagustín, M. (2018). Tools to Support Self-Regulated Learning in Online Environments: Literature Review. En V. Pammer-Schindler, M. Pérez-Sanagustín, H. Drachsler, R. Elferink, y M. Scheffel (Series Eds.), Lecture Notes in Computer Science. Lifelong Technology-Enhanced Learning. EC-TEL 2018. Lecture Notes in Computer Science (Vol. 11082, pp. 16-30). Cham: Springer.

Pérez-Álvarez, R., Maldonado-Mahauad, J. J., Sapunar-Opazo, D., y Pérez-Sanagustín, M. (2017). NoteMyProgress: A tool to support learners' self-regulated learning strategies in MOOC environments. En E. Lavoué, H. Drachsler, K. Verbert, J. Broisin, y M. Pérez-Sanagustín (Eds.), Data Driven Approaches in Digital Education. EC-TEL 2017. Lecture Notes in Computer Science (Vol. 10474, pp. 460-466). Cham: Springer. https://doi.org/10.1007/978-3-319-66610-5_43

Powell, S., y Yuan, L. (2013). MOOCs and open education: Implications for higher education. Descargado de https://publications.cetis.org.uk/wp-content/uploads/2013/03/ MOOCs-and-Open-Education.pdf

Schunk, D. H., y Zimmerman, B. J. (1997). Social origins of self-regulatory competence. Educational Psychologist, 32(4), 195-208. https://doi.org/10.1207/s15326985ep3204_1

Tschofen, C., y Mackness, J. (2012). Connectivism and dimensions of individual experience. The International Review of Research in Open and Distributed Learning, 13, 124-143. https://doi.org/10.19173/irrodl.v13i1.1143

Volet, S., Summers, M., y Thurman, J. (2009). High-level co-regulation in collaborative learning: How does it emerge and how is it sustained? . Learning and Instruction, 19(2), 128-143. https://doi.org/10.1016/j.learninstruc.2008.03.001

Wirth, J. (2009). Guest Editorial: Promoting self-regulated learning through prompts. German Journal of Educational Psychology, 23, 91-94. https://doi.org/10.1024/1010-0652.23.2.91

Zimmerman, B. J. (2000). Attaining Self-regulation: A Social Cognitive Perspective. En M. Boekaerts, P. Pintrich, y M. Zeidner (Eds.), Handbook of Self-Regulation (pp. 13-39). San Diego, CA, US: Academic Press. Handbook of self-regulation. 